\title{
Trastornos pódales en bovinos de sistemas de producción doble propósito en el Departamento Córdoba, Colombia
}

\author{
Disorders hoof in cattle systems of dual production purpose in the Córdoba \\ Department, Colombia
}

Álvarez P Jaime ${ }^{1 *}$ M.Sc; Martínez M Mastoby ${ }^{12}$ M.Sc; Cardona A José ${ }^{13}$ PhD.

${ }^{1}$ Universidad de Córdoba, Facultad de Medicina Veterinaria y Zootecnia, Departamento de Ciencias Pecuarias, Montería, Colombia.

${ }^{2}$ Grupo de investigación MECIVET.

${ }^{3}$ Grupo de Investigación en Medicina de Grandes Animales (MEGA).

\section{Keywords:}

Podology;

hoof;

lameness;

(Source: MeSh).

\section{Palabras Clave:}

Podología;

pezuña;

claudicación;

(Fuente: MeSh).
INFORMACIÓN

Recibido: 29-12-2016;

Aceptado: 01-05-2017.

Correspondencia autor:

jalvarezpt@yahoo.com

\begin{abstract}
The objetive was determining the frequency of pedal upheavals in double purpose bovines in the Córdoba department. A study was done of cross section using 170 greater cows of three year selected at random in different property which were evaluated by means of the accomplishment of the clinical examination of the hooves, determining normality or then abnormality that these displayed and in agreement with the found injury, it was identified and characterized. The frequency of pedals upheavals in Córdoba department was of $64.70 \%$ of the total of the studied sample. $30.59 \%$ of the studied animals displayed alteration of the relation $2: 1$ wall-heel and $47.05 \%$ displayed some type of podal pathology. The found pedals pathologies were: Hoof in corkscrew (36.47\%), digital granuloma (17.06\%), septic pododermatitis $(4.71 \%)$ and interdigital callus $(2.35 \%)$ the incidence of lameness was of $78.75 \%$ with respect to the animals that displayed pathologies and $37.05 \%$ with relation to the total of the sample. In addition was dependency between the type of land with relation to the podal upheaval and independence between podales adjustments and presence of footbaths with relation to the podal adjustment.
\end{abstract}

\section{Resumen}

El Objetivo fue determinar la frecuencia de trastornos pódales en bovinos doble propósito en el Departamento de Córdoba. Se hizo un estudio de corte transversal utilizando 170 vacas mayores de tres años escogidas al azar en diferentes fincas del departamento de Córdoba, Colombia, las cuales fueron evaluadas mediante la realización del examen clínico general y del sistema locomotor, con énfasis en la evaluación de las pezuñas, determinando la normalidad o anormalidad que presentaron estas y de acuerdo con la lesión encontrada se identifico dicho trastorno. La frecuencia de trastornos pódales en el Departamento de Córdoba fue del $64,70 \%$ del total de la muestra estudiada. El 30,59\% de los animales estudiados presentaron alteración de la relación 2:1 muralla-talón y el 47,05\% presentaron algún tipo de patología podal. Las patologías pódales encontradas fueron: Pezuña en tirabuzón (36,47\%), Granuloma digital (17,06\%), Pododermatitis séptica $(4,71 \%)$ y Callo interdigital (2,35\%). La incidencia de cojeras fue del $78,75 \%$ con respecto a los animales que presentaron patologías y de 37,05\% con relación al total de la muestra. Además se encontró dependencia entre el tipo de terreno con relación al trastorno podal e independencia entre arreglos pódales y presencia de pediluvios con relación al trastorno podal. 


\section{Introducción}

Los trastornos podales se definen como toda alteración presente en la pezuña bovina, ya sea de tipo estructural, funcional o infeccioso, que puede o no cursar con claudicación, es así como en muchas explotaciones ganaderas se han detectado grandes pérdidas económicas atribuidas a la presencia de alteraciones digitales en el ganado, así como las ocasionadas por las enfermedades neonatales, las mastitis y las alteraciones reproductivas (CARDONA y CANO 2003; NICOLETTI et ál. 2001; ORREGO et ál. 2003).

Las enfermedades podales influencian el bienestar de los bovinos, promoviendo cambios de conducta en el periodo de pastoreo y rumia, descanso, período de decúbito y tiempo y cantidad de ingesta de agua (SILVA et ál. 2006; SILVA et ál. 2013a). Por lo que influyen negativamente en la producción, aumentando las pérdidas en la condición corporal, disminuyendo el desarrollo reproductivo, la producción de leche y carne, aumentando el número de enfermedades de la glándula mamaria, de igual forma aumenta el descarte prematuro de animales con buena producción o de alto valor genético, así como el aumento de los gastos de medicamentos y mano de obra (FERREIRA et ál. 2005; SILVA et ál. 2001; SILVA et ál. 2007).

Los animales con problemas digitales, entran en un proceso de disconfort, lo que conlleva a pérdidas de condición corporal por la disminución del consumo voluntario de alimento, debido a que tienen dificultades para desplazarse a los sitios de alimentación o pastorear los mejores piensos, pudiendo causar trastornos reproductivos, así como pérdidas en la productividad per cápita de leche entre el 20 y $25 \%$ y de carne aproximadamente en un $10 \%$, también se plantea una reducción de la natalidad mayor al $15,0 \%$. En el caso de los machos, ocasiona disminución en la producción de la cantidad y calidad de semen eyaculado y la duración de vida de los espermatozoides, además disminuye el potencial de salto especialmente cuando las lesiones más dañinas están en las extremidades posteriores y con ello la vida productiva del toro (FERREIRA et ál. 2004; MELENDEZ et ál. 2003; SILVA et ál. 2013b; SOUZA et ál. 2006).

RIERA-NIEVES et ál. (2011), expresan que las características de conformación de las pezuñas, junto con el ambiente y el manejo, constituyen factores predisponentes muy importantes para la incidencia de problemas pódales. Mientras que MOURA et ál. (2010), manifiesta que las causas que predisponen a los animales a padecer trastornos podales pueden ser de origen metabólico, mecánico e infeccioso, y que clínicamente, las enfermedades digitales pueden presentar reacciones proliferativas, infecciosas o ulcerativas localizadas en los cascos, espacio interdigital y borde coronario, siendo que la dermatitis interdigital está presente en varios establos.
Existe una variedad de lesiones digitales asociadas a la claudicación que están relacionadas con la presencia de agentes infecciosos, es así como el tipo de lesión puede varias de acuerdo con el tipo y grado de la enfermedad; la severidad muchas veces es atribuida a la extensión de la lesión y al comprometimiento de las estructuras que componen el estrato córneo. Así sea que clínicamente, una lesión que muestre tener un comprometimiento de tejidos leve o moderado son consideradas como causantes de claudicaciones graves en bovinos (RABELO et ál. 2009).

De igual forma, las infecciones de la cavidad sinovial distal son causas comunes de deformidad de la pezuña en bovinos, siendo la articulación interfalángica distal y la vaina digital tendínea las más afectadas, especialmente cuando ocurren heridas perforantes en las regiones proximal o distal de los dígitos (KOFLER y MARTINEK 2005). Las enfermedades metabólicas, tales como la acidosis láctica ruminal, laminitis y carencias extremas de ciertos nutrientes, pueden promover alteraciones en la calidad y en el crecimiento de las pezuñas. La presencia de tejido córneo de mala calidad constituye un factor predisponente para el desarrollo de otros procesos infecciosos o degenerativos futuros, que generarán claudicación en los bovinos comprometidos (OLLHOFF y ORTOLANI 2001). Los trastornos podales de los bovinos son más comunes en explotaciones ganaderas con sistemas de producción intensivo con instalaciones de piso de cemento, con acúmulo de purines (materias fecales y orina), asociados a errores en la alimentación y a la ausencia de medidas preventivas (FERREIR et ál. 2004; MARTINS et ál. 2002).

La finalidad de este trabajo fue estudiar la frecuencia de presentación de alteraciones podales en bovinos doble propósito, en condiciones de manejo de pastoreo rotacional y semi-extensivo, en fincas del Departamento de Córdoba, Colombia.

\section{Materiales y métodos}

Se realizó un estudio de tipo descriptivo prospectivo de corte transversal, para el cual se eligieron diferentes sistemas de producción de bovinos de doble propósito. Fue realizado en el departamento de Córdoba (Colombia), ubicado entre los coordenadas $7^{\circ} 23^{\prime}$ y $9^{\circ} 26^{\prime}$ de latitud norte y los $74^{\circ} 52^{\prime}$ y $76^{\circ} 32^{\prime}$ de longitud al oeste del meridiano de Greenwich, a una altura de 30 m.s.n.m, con temperatura promedio anual de $28^{\circ} \mathrm{C}$, humedad relativa del $82 \%$, precipitación media anual de $1400 \mathrm{~mm}$ y pertenece a la formación climática de bosque tropical lluvioso, se presentan dos estaciones bien definidas (época de lluvia y época seca) (PABÓN et ál. 2001).

Para la escogencia de las explotaciones y de los animales se utilizó el universo total de animales suministrados por la Coordinación Fedegan - Fondo Nacional Del Ganado Censo de Vacunación de 
Noviembre a Diciembre de 2010. El tamaño de la muestra fue de 170 animales, usando un error máximo permisible del $9 \%$, una confiabilidad de $98 \%$ y una prevalencia estimada del $70 \%$, los datos obtenidos fueron digitados en una hoja electrónica de Excel y analizados en software estadístico S.A.S 9.1.3 (SAS 2007), para las variables de tipo descriptivo y de tipo cualitativo se realizaron tablas de contingencia que permitieron relacionar la presencia o no de patologías pódales con algunas observaciones realizadas De igual forma fue evaluada la independencia estadística con la prueba $X^{2}$ (LOHR, 2000).

Para la recolección de las muestras, los animales no se sometieron a dolor y/o estrés innecesario, por lo que fueron inmovilizados tomando en cuenta las normas técnicas en el manejo y sujeción de animales, enmarcado en el cumplimiento de la Declaración Universal de los Derechos de los Animales, referente a los principios éticos internacionales para la investigación biomédica con animales del CIOMS (Council for International Organizations of Medical Sciences) establecida por la UNESCO (United Nations Educational, Scientific and Cultural Organization) y la OMS (Organización Mundial de la Salud) en 1949 y de la Ley 84 de Octubre 27 de 1989 (Estatuto Colombiano de Protección Animal) (MRAD, 2006), por lo que fueron evaluados por medio de examen clínico físico general y previa sujeción, derribe del animal y asepsia, se procedió a la limpieza o blanqueo de las pezuñas, analizando y caracterizando las lesiones encontradas, así como la determinación de los parámetros anatómicos de la misma. Posteriormente fueron tomadas fotografías de alta definición (Sony DSC H10, Japón; Nikon D7000, Japón) para su posterior análisis en computador (HP ENVY dv6 Notebook PC, China).

\section{Resultados y discusión}

En la Figura 1, se puede observar la relación de trastornos pódales encontrados, donde el $64,70 \%$ (110/170) presentaron algún tipo de trastorno podal, de los cuales el 47,06\% (80/170), presentó algún tipo de lesión podal, el 17,65 \% (30/170) presentaron defectos de conformación principalmente en la relación 2:1 muralla-talón (pezuñas largas), el 12,94\% (22/170), presentaron defectos de conformación y patologías podales y el $37,05 \%$ (63/170) presentaron claudicación. Estos resultados son parecidos a los reportados por BENAVIDES et ál. (2012), quienes informaron una prevalencia del $82,05 \%$ en el Departamento de Nariño (Colombia). Así como SILVEIRA et ál. (2011) y QUEIROZ et ál. (2011), quienes reportaron incidencias de vacas afectadas con trastornos podales del $82,6 \%$ y $86,43 \%$ en Pará y Paraná (Brasil) respectivamente, prevalencias inferiores fueron reportadas por FLOR y TADICH (2008) y SILVEIRA et ál. (2009), con $28,7 \%$ y
$22,25 \%$ en vacas lecheras del sur de Chile y del estado de Pará (Brasil) respectivamente, mientras que TADICH et ál. (2005), CONFALONIERI et ál. (2008), MACHADO et ál. (2008) y TOMASELLA et ál. (2014) encontraron que el $9,1 \%, 2,71 \%, 5,08 \%$ y $8,5 \%$ de los animales de sus estudios en el Sur de Chile, Tandil (Argentina), Maranhão y Belo-Horizonte (Brasil) respectivamente presentaron alteraciones podales.

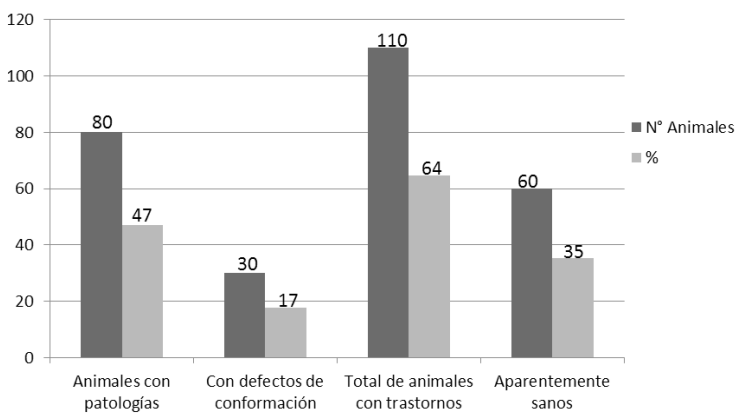

Figura 1. Relación de trastornos podales encontradas en 170 bovinos de doble propósito en el departamento de Córdoba, Colombia.

En la Tabla 1, se muestra la descripción de patologías pódales encontradas y su respectivo porcentaje, de las cuales el $47,05 \%(80 / 170)$ presentaron algún tipo de patología podal, entre estas el $34,11 \%$ (58/170) presentaron una patología, 12,35\% (21/170) presentaron dos patologías y solo 1 animal $(0.58 \%)$ presento tres patologías conjuntas. Entre las patologías pódales encontradas, la pezuña en tirabuzón fue la de mayor prevalencia con $36,47 \%$ (62/170), seguido del granuloma digital con $17,06 \%(29 / 170)$, la pododermatitis séptica con $4,71 \%(8 / 170)$ y el callo interdigital con 2,35\% (4/170). Este resultado concuerda con lo informado por SOUZA et ál. (2007), quienes reportaron una prevalencia del $24,1 \%$ de pezuñas en tirabuzón, atribuyéndoselo a la escasa utilización de arreglos funcionales de pezuña en las explotaciones de su estudio, así mismo TADICH et ál. (2005), informaron como mayor causante de claudicación las deformaciones crónicas de la pezuña con 19,8\%. Una explicación a la elevada prevalencia en el presente estudio podría ser los desórdenes nutricionales a los que se ve enfrentada la vaca durante su vida productiva que puede conllevar a laminitis crónicas, así como es el reflejo de la poca preocupación de los propietarios sobre el cuidado rutinario de la pezuña.

De igual forma, SILVEIRA et ál. (2009), informaron en su estudio que la patología más frecuente fueron los fibromas o hiperplasias interdigitales $(80,9 \%)$, pudiendo deberse a reacciones proliferativas con hiperplasia de tejido fibroso denso de la piel interdigital y tejido subcutáneo que sucede en respuesta a la irritación crónica subsecuente a traumas o condiciones 
Tabla 1. Descripción de patologías pódales encontradas en 80 bovinos de doble propósito en el departamento de Córdoba, Colombia.

\begin{tabular}{|c|c|c|c|c|}
\hline Patologías & Miembros Afectados & $\mathbf{N}^{\circ}$ Animales & $\begin{array}{l}\% \text { del total con } \\
\text { Patologías (80) }\end{array}$ & $\begin{array}{c}\text { \% del total } \\
\text { Muestra (170) }\end{array}$ \\
\hline \multirow{4}{*}{ Pezuña en Tirabuzón } & Anteriores & 11 & 13,75 & 6,47 \\
\hline & Posteriores & 42 & 52,50 & 24,71 \\
\hline & Ambos & 9 & 11,25 & 5,29 \\
\hline & Total & 62 & 77,50 & 36,47 \\
\hline \multirow{4}{*}{ Granuloma Digital } & Anteriores & 6 & 7,50 & 3,53 \\
\hline & Posteriores & 20 & 25,00 & 11,76 \\
\hline & Ambos & 3 & 3,75 & 1,76 \\
\hline & Total & 29 & 36,25 & 17,06 \\
\hline \multirow[t]{4}{*}{ Podo Dermatitis Séptica } & Anteriores & 2 & 2,50 & 1,18 \\
\hline & Posteriores & 4 & 5,00 & 2,35 \\
\hline & Ambos & 2 & 2,50 & 1,18 \\
\hline & Total & 8 & 10,00 & 4,71 \\
\hline \multirow{4}{*}{ Callo Iterdigital } & Anteriores & 1 & 1,25 & 0,59 \\
\hline & Posteriores & 2 & 2,50 & 1,18 \\
\hline & Ambos & 1 & 1,25 & 0,59 \\
\hline & Total & 4 & 5,00 & 2,35 \\
\hline
\end{tabular}

ambientales con acúmulo de deyecciones y humedad excesiva, como pudo suceder con los animales del estudio ya que el granuloma interdigital con 17,06 $\%(29 / 170)$, fue la patología con la segunda mayor prevalencia.

Sin embargo, los resultados del presente estudio difieren con los reportados por RABELO et ál. (2013), quienes encontraron dermatitis digital $(23,7 \%)$, pododermatitis séptica $(12,6 \%)$, doble suela $(12,1)$ dermatitis interdigital $(7,9 \%)$ en el estado de Goías. TOMASELLA et ál. (2014), encontraron más frecuente la úlcera de suela $(51,47 \%)$, seguida de enfermedad de la línea blanca $(25,74 \%)$ y sula fina o delgada $(10,29 \%)$ en Belo-Horizonte (Brasil). Así mismo BENAVIDES et ál. (2012), reportaron erosión del talón $(53,84 \%)$, seguida de hemorragia de la suela $(46,15 \%)$, separación de línea blanca $(28,20 \%)$ y amarillamiento de la suela $(23,07 \%)$ en bovino lecheros de Nariño (Colombia). SILVA et ál. (2011), informaron frecuencias de erosión de talón $(8,3 \%)$, pododermatitis séptica $(13,9 \%)$, dermatitis digital $(11,0 \%)$ y dermatitis interdigital $(4,2 \%)$ en el estado de Goías (Brasil). QUEIROZ et ál. (2011) encontraron en su estudio que la principal lesión podal fue la erosión de talón $(50,66 \%)$, seguida de pinza larga/talón bajo $(21,29 \%)$ y suela plana $(8,29 \%)$ en vacas confinadas en el estado de Paraná (Brasil). CONFALONIERI et ál. (2008), reportaron que las principales patologías observadas fueron la dermatitis interdigital $(29,79 \%)$, dermatitis digital $(25,53 \%)$ y laminitis crónica $(20,06)$ en Tandil (Argentina), así mismo, FLOR y TADICH (2008), reportaron enfermedad de la línea blanca $(82,5 \%)$, erosión de talones $(53,3 \%)$, hemorragia plantar $(24,6 \%)$, doble suela $(13,7 \%)$, ulcera plantar $(7,8 \%)$ y dermatitis digital $(6,6 \%)$ al evaluar rebaños pequeños del sur de Chile. Finalmente SILVA et ál. (2004), en su estudio realizado en bovinos del estado de Goiás (Brasil), informaron que la dermatitis digital fue observada en $36,82 \%$ de los animales, seguida de la pododermatitis necrosante $(32,95 \%)$ y de la pododermatitis interdigital vegetativa $(7,5 \%)$ fueron las más prevalentes. Todas las variaciones con los diferentes estudios, podrían estar influenciadas por las diferentes características raciales, condición corporal, edad, manejo profiláctico y nutricional, así como el ambiente, tipo de explotación y el terreno donde pastan los animales.

En la Figura 2, su puede observar el número de animales con claudicación dependiendo de la patología, así como el porcentaje del total de animales con patologías podales y del total de la población examinada. De los cuales, el $78,75 \%$ (63/80) de los animales que presentaron patologías evidenciaron algún grado de claudicación, correspondiente al $37.05 \%$ (63/170) del total de la muestra. Resultados superiores a investigaciones realizadas por RIERANIEVES et ál. (2011), quienes informaron un $8,49 \%$ de vacas con lesiones en su estudio, de las cuales $4,15 \%$ presentaron claudicación leve y el 3,02\% moderada. CONFALONIERI et ál. (2008), reporto en animales con lesiones podales, cojeras grado 3 superiores al $60 \%$ y cojeras grado 4 del $22 \%$. Sin embargo, DYER et ál. (2007) manifiestan que más de un tercio de las vacas con lesiones de pezuña no muestran signos obvios de alteración de su locomoción. La prevalencia de claudicaciones del presente estudio $(37,05 \%)$, se considera como valores estimados anormales, ya que prevalencias mayores al $15 \%$ en una explotación deben causar alarma y se deben buscar las causas para aplicar los correctivos (BOOTH et ál. 2004), sin embargo la prevalencia del presente estudio pudo ser superior, ya que no fueron consideradas las graduaciónes de las claudicaciones, por lo que lo más probable es que los investigadores desestimaran los grados 1 y 2 como los más leves. 


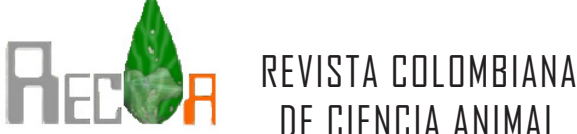

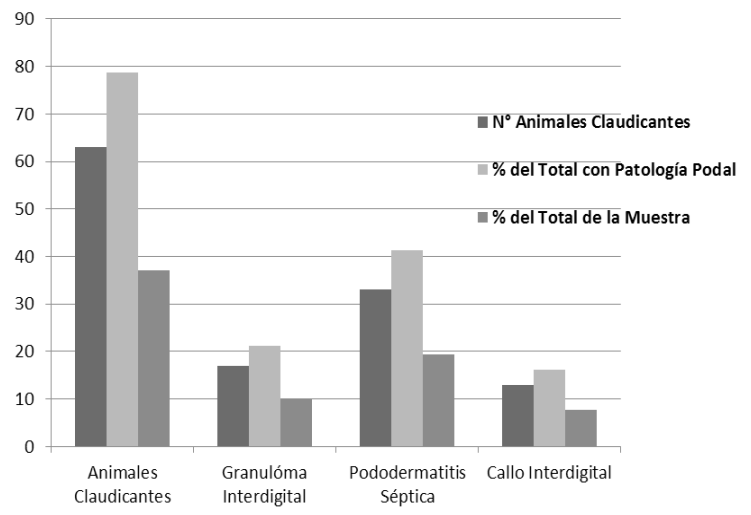

Figura 2. Número y porcentaje de animales con claudicación entre los animales con patología y del total de la población según las patologías más frecuentes.

La patología que presento mayor número de animales claudicantes, fue el granuloma interdigital con un $21,25 \%(17 / 80)$ del total de animales con patologías, correspondientes a un $10 \%(17 / 170)$ del total de la muestra, seguido de la pododermatitis séptica y el callo interdigital (Figura 3). Mientras que CONFALONIERI et ál. (2008) reportaron como mayor causante de claudicaciones en su estudio a la dermatitis digital $(29,79 \%)$. Sin embargo TADICH et ál. (2005) reportaron como mayor causante de claudicación en los animales de su estudio a la deformaciones crónicas de la pezuña $(19,8 \%)$ seguida de las lesiones de la línea blanca (19,0\%), así mimo Flor y TADICH (2008), informaron mayor presentación de enfermedad de la línea blanca $(82,5 \%)$ seguida de erosión de talones $(53,3 \%)$ en las vacas cojas de su estudio.

En la Figura 4, se puede observar el número y porcentaje de animales con pezuñas largas (alteración en la relación 2:1 muralla-talón), de las cuales el 30,59\% (52/170) del total de animales muestreados presentaron la alteración, de estos el 44,23\% (23/52) presentaron algún tipo de patología podal correspondientes al $13,53 \%(23 / 170)$ del total de la muestra. Esto tal vez se debe al desequilibrio entre el crecimiento y el desgaste de la pezuña, debido a que por las condiciones de humedad excesiva del terreno se disminuye el desgaste, favoreciendo el crecimiento de la pezuña, por lo que se desvía el apoyo hacia los talones. Es así como MOLINA et ál. (1999), indican que los factores ambientales y las instalaciones están directamente relacionadas con el grado de claudicación causando desgaste y

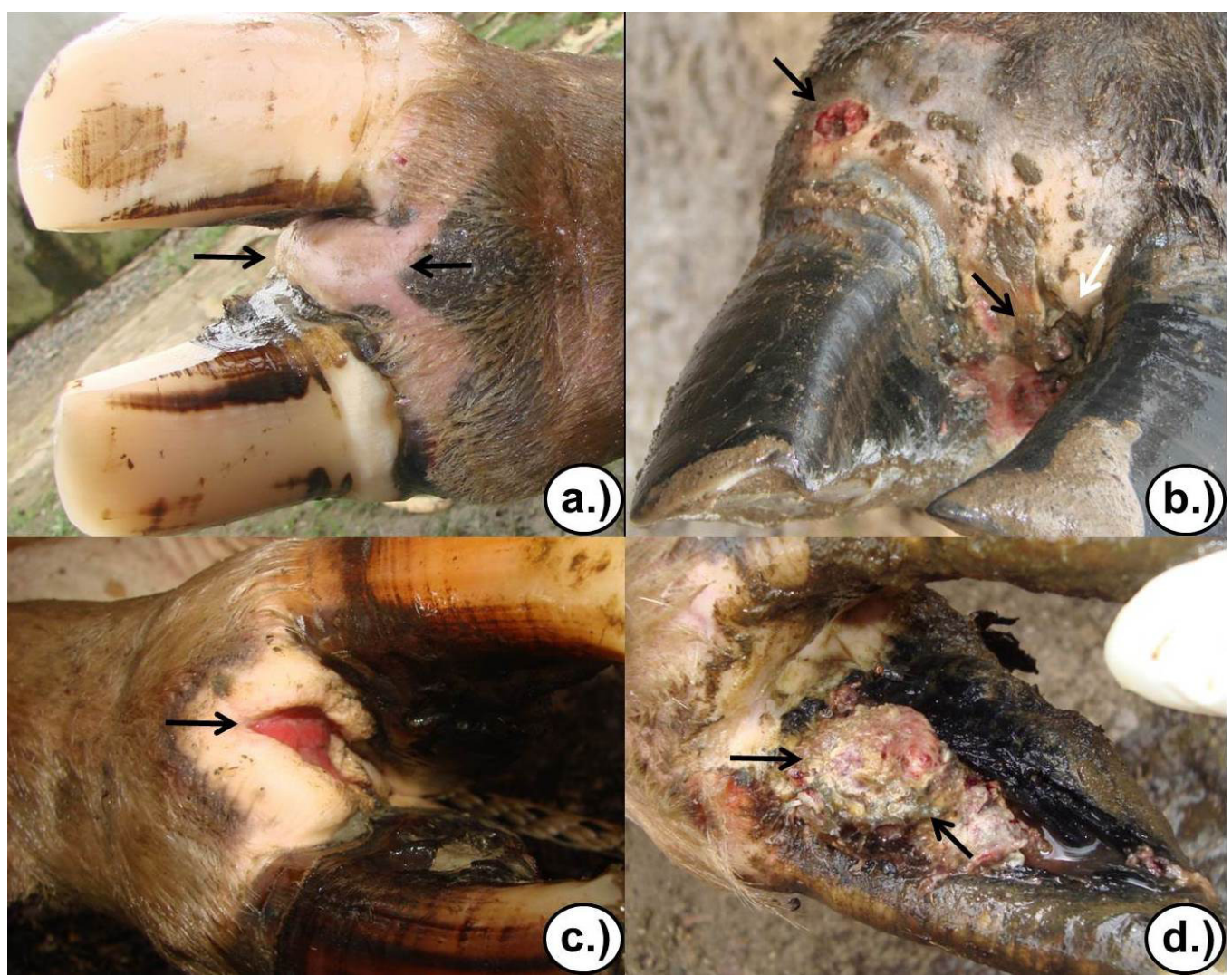

Figura 3. Lesiones podales en bovinos doble propósito del departamento de Córdoba. a.) Callo interdigital (flechas). b.) Pododermatitis séptica (flechas). c.) Necrobacilosis interdigital (flecha). d.) Granuloma interdigital (flechas). 
ablandamiento excesivo de los dígitos debido al tipo de piso o al exceso de humedad, de igual manera OLLHOFF y ORTOLANI, (2001), explican que ese equilibrio entre desgaste y crecimiento está influenciado por el sistema de manejo, ya sea en confinamiento o pastoreo, sin embargo, aclaran que no existe diferencia racial en cuanto al crecimiento y desgaste de los cascos. MACHADO et ál. (2008), constató que las instalaciones que poseen pisos de cemento que en la mayoría de las veces es desgastado y consecuentemente abrasivo lo que ocasiona desgaste del tejido corneo.

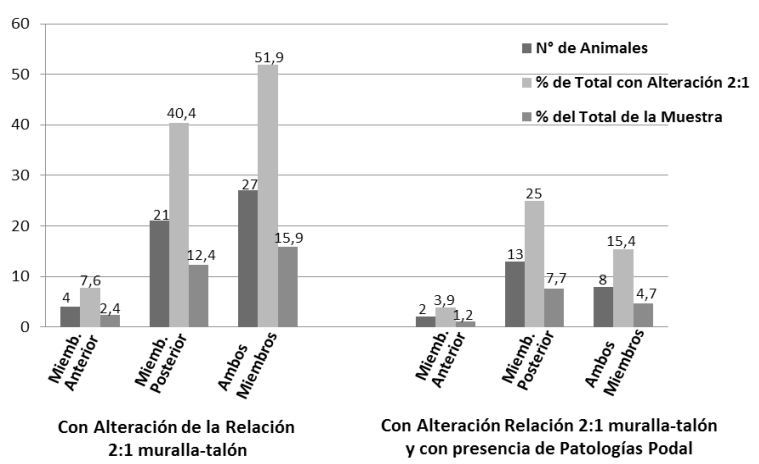

Figura 4. Animales que presentaron pezuñas largas (alteración de la relación 2:1 muralla-talón).

Al caracterizar los miembros por separado, se puede percibir que los posteriores son los más afectados por este tipo de alteración, siendo el 40,38\% (21/52) de los animales con alteración de la relación 2:1, correspondientes al 12,35\% (21/170) del total de la muestra. El 25,0\% (13/52) de los animales con pezuñas largas (alteración en la relación 2:1 murallatalón) presentaron además alguna patología podal, equivalente al $7,65 \%(13 / 170)$ del total de la muestra. Estos datos concuerdan con los reportados por CONFALONIERI et ál. (2008), FLOR y TADICH (2008), OLLHOFF y ORTOLANI (2001) y TADICH et ál. (2005), quienes reportaron en sus estudios mayor presentación de alteraciones digitales en los miembros posteriores, posiblemente porque éstos soportan mayor peso, contribuyendo a un mayor desgaste del tejido córneo en esa región.

En las figuras 5,6 y 7 se analizan la presentación de trastornos podales según algunas condiciones de manejo de las diferentes explotaciones ganaderas del departamento de Córdoba, como fueron la presencia de pediluvios, el arreglo funcional de pezuñas y el tipo de terreno donde pastaban los animales del estudio. Es así como el $14,71 \%$ (25/170) de los animales eran manejos con sistemas de pediluvio, de ellos el 9,41\% (16/170) presentaron trastornos podales. Así mismo, el 45,88\% (78/170) recibieron arreglos funcionales de pezuña, de los cuales el 20,0\% (34/170) presento trastorno podal. Mientras que, respecto al tipo de potrero el $81,76 \%$
$(139 / 170)$ pastaban en terrenos bajos o anegadizos, de los cuales el 42,35\% (72/170) presentaron trastornos podales.

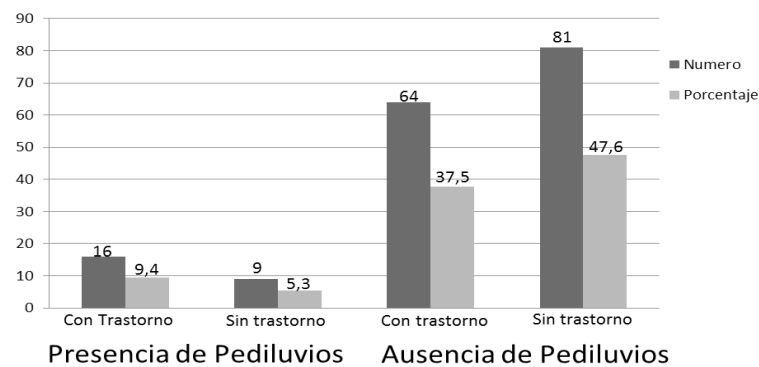

Figura 5. Relación entre la presencia de trastorno podal y la uso de pediluvios en las explotaciones del Departamento de Córdoba.

Al realizar la prueba de Chí Cuadrado (0.066), se determinó que no había dependencia estadística significativa $(P>0.05)$, por lo que la presencia de pediluvios no influye en la presentación y o control de trastornos podales en los animales estudiados, pudiendo deberse a que tengan pediluvios en las explotaciones pecuarias, pero no eran usados adecuadamente o con productos o soluciones mal manejadas. Este resultado contrasta con el reportado por HETTICH et ál. (2007), quienes encontraron que si existía asociación positiva estadísticamente significativa entre la presencia y uso de pediluvios y el control de patologías podales en rebaños del sur de Chile. PERUSIA (2001), recomienda el uso de pediluvios cada 20 a 25 días debido a su efecto endurecedor del casco y antiséptico, de esta manera actúa protegiendo al pododermo de las invasiones bacterianas, principalmente en períodos de excesiva humedad. MÜNZENMAYER (1997), manifiesta que los pediluvios bien utilizados constituyen una herramienta que contribuye en el tratamiento y control de las afecciones podales principalmente de carácter infeccioso y necrótico-corrosivas del bovino

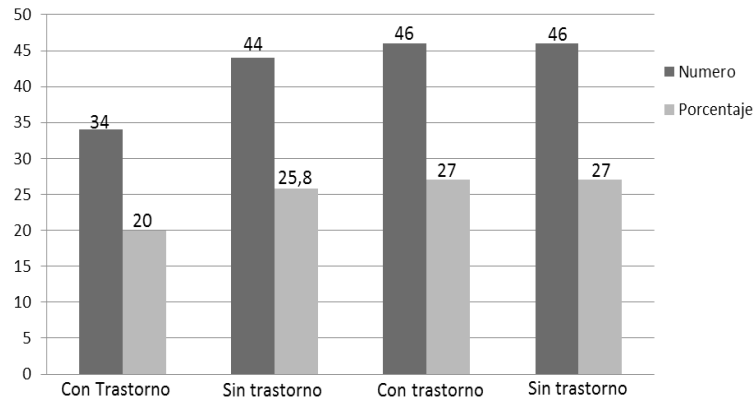

Con Arreglos Funcionales Sin Arreglos Funcionales

Figura 6. Relación entre la presencia de trastorno podal y la realización de arreglos funcionales de pezuña en las explotaciones del Departamento de Córdoba. 
Al realizar la prueba de Chí Cuadrado (0.404), se determinó que no había dependencia estadística significativa $(P>0.05)$, por lo cual se concluye la realización de arreglo funcional de pezuña en los bovinos del estudio no influye en la tasa de presentación de trastornos pódales, pudiendo deberse al mal arreglo de las pezuñas por parte de la persona encargada de realizarlos, ya sea por inexperiencia o falta de capacitación, así como la posible realización del procedimiento a animales con problemas podales subclínicos, en los cuales el procedimiento aumentaría el grado de dolor. Sin embargo, contrasta con lo reportado por GARCÍA-BRACHO (2009), quienes si encontraron asociación estadísticas significativas en su estudio, en el cual se observó que de los animales que recibieron arreglos funcionales o quiropedia, solo enfermaron el $4 \%$ de ellos, mientras que los animales del grupo control enfermaron el 45,4\%. GREENOUGH (2001), manifiesta que los arreglos funcionales disminuyen la presentación de alteraciones anatómicas como la pezuña en tirabuzón y las fisuras y sobre crecimientos del estrato córneo.

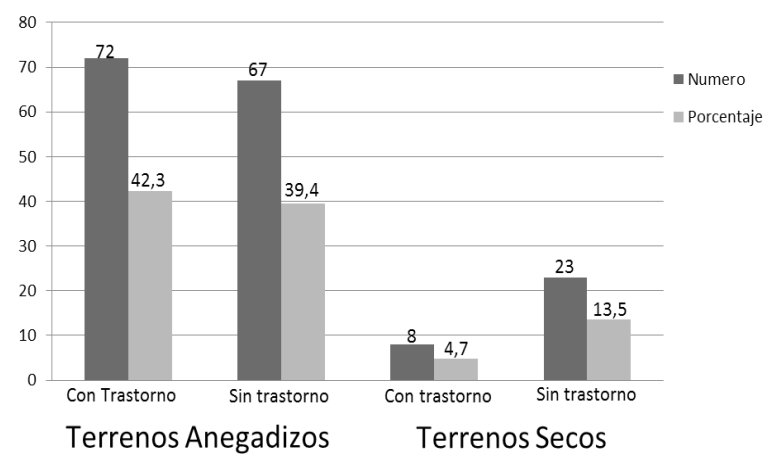

Figura 7. Relación entre la presencia de trastorno podal y el tipo de terreno donde pastaban los animales en las explotaciones del Departamento de Córdoba.

Al realizar la prueba de Chí Cuadrado (0.008), se determinó que si hubo dependencia estadística significativa $(P<0.05)$, por lo que se concluye que el tipo de terreno donde pastan los animales (terreno anegadizo o terreno seco) influye positivamente en la presentación de trastornos pódales en los bovinos estudiados. Este resultado concuerda con el informado con CONFALONIERI et ál. (2008), quienes encontraron que en los establecimientos con suelo no deseable, las vacas tienen 2.58 veces más chances de presentar patologías podales que en los establecimientos con suelo deseable. MAUCHLE et ál. (2008), explican que la elevada humedad del terreno, posiblemente propicia el reblandecimiento del estrato córneo de las pezuñas, tornándose más susceptibles a infecciones bacterianas, como es el caso de la erosión de talón y los traumatismos que causan lesiones como hemorragia de suela, enfermedad de la línea blanca y doble suela y que aumentan en los periodos lluviosos debido al mayor acúmulo de agua en terrenos bajos o inundables sumado a los pastos sucios y que al ser llevados al corral de ordeño tiene que desplazarse por caminos escabrosos o pedregosos, lo que puede aumentar la ocurrencia de traumatismos y consecuentemente lesiones hemorrágicas en la suela de los dígitos.

\section{Conclusiones}

Del presente estudio se puede concluir que se obtuvo una prevalencia de trastornos pódales en el departamento de Córdoba del 64,70\%. El 30,59\% de los animales estudiados presentaron pezuñas largas (alteración de la relación 2:1 muralla-talón), el $47,05 \%$ presentaron algún tipo de patología podal y el $37,05 \%$ presentaron claudicación. Las patologías pódales encontradas con mayor presentación fueron las pezuñas en tirabuzón, seguida de el granuloma digital, la pododermatitis séptica y el callo interdigital. Los miembros más afectados con pezuñas largas (alteración de la relación 2:1 muralla-talón) fueron los posteriores. La presencia de pediluvios y el arreglo funcional de la pezuña bovina no influyeron en la tasa de presentación de trastornos pódales, mientras que el tipo de terreno en el que pastaban si influyó positivamente. Es escasa la información que existe sobre diferentes aspectos de la podología en bovinos de doble propósito, por lo que se hace necesario continuar con la realización de investigaciones tendientes a generar datos que ayuden a disminuir la incidencia de problemas que influyan negativamente en los sistemas productivos.

\section{Referencias}

BENAVIDES, E.; MARTINEZ, F.; QUEVEDO, D. 2012. Prevalencia de lesiones asociadas con laminitis subclinica en vacas holstein de primer parto en Nariño, Colombia. Rev Inv Pec 1(1):63-70.

BOOTH, C.; WARNICK, L.; GRÖHN, T.; MAIZON, D.; GUARD, C.; JANSSEN, D. 2004. Effect of lameness on culling in dairy cows. J Dairy Sci. 87(12): 4115-22.

CARDONA, J.; CANO, N. 2003. Alteraciones digitales en el ganado bovino del trópico bajo. MVZ-Córdoba. 8:(1):249-253. 
CONFALONIERI, O.; SORACI, A.; PASSUCCI, J.; RODRÍGUEZ, E.; BECALUBA, H.; TAPIA, M. 2008. Prevalencia y detección de factores de riesgo de patologías podales y su influencia en la producción láctea en bovinos de la cuenca lechera mar y sierras de Tandil. Analecta Veterinaria. 28(1):15-20.

DYER, R.; NEERCHAL, N.; TASCH, U.; WU, Y.; DYER, P.; RAJKONDAWAR, P. 2007. Objective determination of claw pain and its relationship to limb locomotion score in dairy cattle. J Dairy Sci 90:4592-4602.

FERREIRA, P.; LEITE, R.; CARVALHO, A.; FACURY, E.; SOUZA, R.; FERREIRA, M. 2004. Custo e resultados do tratamento de seqüelas de laminite bovina: relato de 112 casos em vacas em lactação no sistema free-stall. Arq. Bras. Med Vet Zootec 56(5):589-594.

FERREIRA, P.; CARVALHO, A.; FACURY, E.; FERREIRA, M.; FERREIRA, R. 2005. Afecções do sistema locomotor dos bovinos. In: Anais do II Simpósio Mineiro de Buiatria. Belo Horizonte, Brasil. 26p. Disponible en: http://www.ivis. org/proceedings/abmg/2005/pdf04.pdf?LA=7 Accesado el 03/02/2016.

FLOR, E.; TADICH, N. 2008. Claudicaciones en vacas de rebaños lecheros grandes y pequeños del sur de Chile. Arch Med Vet 40:125-134.

GARCÍA-BRACHO, D.; HAHN, M.; PINO, D.; VIVAS, I.; LEAL, M.; CLERC, K. 2009. Prevención de enfermedades podales mediante el recorte funcional de la pezuña al momento del secado en vacas lecheras confinadas en el trópico. Rev. Cient., FCV-LUZ. XIX(2):147-152.

GREENOUGH, P. 2001. Sand cracks, horizontal fissures, and other conditions affecting the wall of the bovine claw. Vet. Clin. N. Am-Food A. 17(1):93-110.

HETTICH, E.; HINOSTROZA, M.; VAN, G.; TADICH, N. 2007. Factores asociados a la presentación de cojeras en 50 rebaños lecheros de la X Región, Chile. Arch. Med. Vet. 39(3):247-253.

KOFLER, J.; MARTINEK, B. 2005. New surgical approach to the plantar fetlock joint through the digital flexor tendon sheath wall and suspensory ligament apparatus in cases of concurrent septic synovitis in two cattle. Vet. J. 169: $370-375$.

MACHADO, P.; PEREIRA, H.; SANTOS, H.; OLIVEIRA, R.; GUERRA, P.; TEIXEIRA, W. 2008. Prevalência e classificação de afecções podais em fêmeas bovinas destinadas à produção de leite na bacia leiteira do município de Itapecuru Mirim-MA. Rev. Bras. Saúde Prod. An. 9 (4): 777 - 786.

MARTINS, C.; SARTI, E.; BUSATO, I.; PIRES, P.; FIORI, C.; MOREIRA, C.; SOARES, K.; BETINI, B.; VELASQUEZ, M. 2002. Prevalência e classificação das afecções podais em vacas lactantes na bacia leiteira de Campo Grande (capital) e municípios arredores - MS. Ens Ciên. 6:113-137.

MAUCHLE, Ú.; CARVALHO, A.; ALZAMORA-FILHO, F.; FERREIRA, P.; FACURY-FILHO, E.; CAVALCANTE, M. 2008. Efeito da sazonalidade sobre a ocorrência de lesões podais em vacas de raças leiteiras. Rev. Bras. Saúde Prod. An. 9(1):109-116.

MELENDEZ, P.; BARTOLOME, J.; ARCHBALD, L.; DONAVAN, A. 2003. The association between lameness, ovarian cyts and fertility in lactating dairy cow. Therogienol. 59(3-4):927-937.

MOLINA, L.; CARVALHO, E.;, FACURY-FILHO, P.; FERREIRA, V. 1999. Prevalência e classificação das afecções podais em vacas lactantes na bacia leiteira de Belo Horizonte. Arq. Bras. Med. Vet. Zoo. 51:149-152.

MOURA, M.; GOULART, D.; ORLANDO, C.; ARÃES, L.; SILVA, O.; SILVA, L. 2010. Dermatite Digital em Bovinos da Raça Nelore: Avaliação do Ganho de Peso, Medidas Testiculares e Epididimárias, no Pós-operatório das Lesões. Vet. Zootec. 17(2):239-249.

MRAD, A. 2006. Ética en la investigación con modelos animales experimentales. Alternativas y las 3 RS de Russel. Una responsabilidad y un compromiso ético que nos compete a todos. Rev. Col. Bioética. 1(1):163-184. 
MÜNZENMAYER, W. 1997. Afecciones podales en rodeos lecheros: desafío profesional. Therios (suppl): 1-31.

NICOLETTI, J.; SOUZA, F.; THOMASSIAN, A.; HUSSNI, C.; ALVES, A. 2001. Prevalência das lesões podais e graus de claudicação em vacas leiteiras mantidas em confinamento permanente ("freestall"e "tie-stall"). Rev Educ Contin. 4:24-32.

LOHR, S. 2000. Muestras de probabilidades simples. En: Muestreo: Diseño y análisis. México: International Thomson Editores, S. A. Pp 30-40.

OLLHOFF, R.; ORTOLANI, E. 2001. Comparação do crescimento e do desgaste do casco em bovinos taurinos e zebuínos. Cienc. Rural. 31(1):67-71.

ORREGO, J.; DELGADO, A.; ECHEVARRÍA, L. 2003. Vida productiva y principales causas de descarte de vacas Holstein en la cuenca de Lima. Rev Inv Vet Perú. 14(1):68-73.

PABÓN, J.; ESLAVA, J.; GÓMEZ, R. 2001. Generalidades de la distribución espacial y temporal de la temperatura del aire y de la precipitación en Colombia. Meteorol. Colomb. 4:47-59.

PERUSIA, O. 2001. Patologías podales del bovino. Rev Inv Vet Perú. 12(2):65-77.

QUEIROZ, G.; PEREIRA, P.; MARCONDES, G.; ROMÃO, F.; ZANLUCHI, A.; SAUT, J.; YAMAMOTO, M.; SILVA, L. 2011. Prevalência de lesões podais em vacas de leite na região norte do Paraná. Vet. Zootec. 18(4 Supl. 3): 340-342.

RABELO, R.; ALVES, J.; LIMA, C.; REIS, E.; BAYLÃO, M.; RODRIGUES, E.; CARVALHO, F.; BANNWART, L. 2009. Identificação e caracterização de diferentes digitais acometendo bovinos leiteiros do município de Jataí-GO e sua influência na claudicação. In: XXXVI Congresso Brasileiro de Medicina Veterinária. Porto Seguro, Brasil. p36.

RABELO, R.; VULCANI, V.; DE SANT'ANA, F.; LIMA, C.; RABBERS, A.; HELRIGEL, P.; DIAS, M. 2013. Influence of different digital diseases in lameness of dairy cows in southwest of the state of Goiás. R. Bras. Ci. Vet. 20(4):198-203.

RIERA-NIEVES, M.; VALS, V.; NIEVES-CRESPO, L.; PÉREZ-AREVALO, M.; GAVIDIA-TERÁN, J.; ZABALETA, J. 2011. Características morfológicas de las pezuñas y su relación con el grado y distribución de las claudicaciónes en vacas de raza carora. AICA. 1:300-303.

SILVA, L.; SILVA, L.; ROMANI, A.; RABELO, R.; FIORAVANTI, M.; SOUZA, T. 2001. Características clínicas e epidemiológicas das enfermidades podais em vacas lactantes do município de Orizona-GO. Ciênc. Na. Bras. 2(2):119-126.

SILVA, L.; FIORAVANTI, M.; TRINDADE, B.; SILVA, O.; EURIDES, D.; CUNHA, P.; SILVA, L.; MOURA, M. 2004. Enfermidades digitais em vacas de aptidão leiteira: associação com mastite clínica, metrites e aspectos epidemiológicos. Pesq Vet Bras. 24:217-222.

SILVA, L.; MORAES, R.; ROMANI, A.; FIORAVANTI, M.; CUNHA, P.; BORGES, J.; MACEDO, S.; DAMASCENO, A.; RABELO, R.; GARCIA, A. 2006. Pododermatite séptica em bovinos: evolução clínica da fase inicial. Braz. J. Vet. Res. An. Sci. 43 (5): $674-680$.

SILVA, L.; SOARES, L.; MOURA, M.; BATISTA, L.; RIBEIRO, G. 2007. Enfermidades digitais em bovinos: efeito do toalete do estojo córneo saudável e enfermo na recuperação dos animais. In: Congresso de Pesquisa, Ensino e Extensão. Goiânia, Brasil. Anais Eletrônicos. [CD-ROM].

SILVA, L.; LIMA, I.; FONSECA, A.; BORGES, N.; FIORAVANTI, M. 2011. Avaliações morfológicas de lesões nas extremidades distais dos membros de bovinos claudicantes. Ci. Anim. Bras. 12(3):566-575.

SILVA, L.; DA CUNHA, P.; NORONHA-FILHO, A.; GOULART, D.; CAMPOS, S.; FREITAS, S. 2013a. Comportamento diário de vacas da raça Girolando com dermatite digital manejadas extensivamente. Vet. Zootec. 20(3):459-466. 
SILVA, D.; MOURA, M.; MIGUEL, M.; DA SILVA, L.; MATOS, M.; MOURA, V. 2013b. Avaliações histológica e histomorfométrica de testículos de bovinos com dermatite digital. Ciênc. Anim. Bras. 14(3):391-398.

SILVEIRA, J.; ALBERNAZ, T.; OLIVEIRA, C.; DUARTE, M.; BARBOSA, J. 2009. Afecções podais em vacas da bacia leiteira de Rondon do Pará. Pesq. Vet. Bras. 29(11):905-909.

SILVEIRA, J.; SILVA, N.; ALBERNAZ, T.; BOMJARDIM, H.; ANDRADE, S.; OLIVEIRA, C.; BARBOSA, J. 2011. Afecções podais em bovinos associado à sodomia. Vet. Zootec. 18(4 Supl. 3):294-297.

SOUZA, R.; FERREIRA, P.; MOLINA, L.; CARVALHO, A.; FACURY-FILHO, E. 2006. Perdas econômicas ocasionadas pelas enfermidades podais em vacas leiteiras confinadas em sistema free stall. Arq. Bras. Med. Vet. Zootec. 58(6):982-987.

SOUZA, R.; CARVALHO, A.; FERREIRA, P.; FACURY, E.; FERREIRA, M.; FERREIRA, R.; COSTA, C.; NETO, A. 2007. Prevalência e distribuição de lesões digitais em vacas leiteiras nas regiões de Belo Horizonte e Pedro Leopoldo. Ciência Animal Brasileira. 8(4):823-831.

STATISTICAL ANALYSIS SYSTEM. SAS OnlineDoc 9.1.3. SAS. Institute Inc, Cary, NC, USA. 2007.

TADICH, N.; HETTICH, E.; VAN SCHAIK, G. 2005. Prevalencia de cojeras en vacas de 50 rebaños lecheros del sur de Chile. Arch. Med. Vet. 37(1):29-36.

TOMASELLA, T.; NEGRI-FILHO, L.; AFFONSO, M.; JUNIOR, F.; DA SILVA, L.; OKANO, W. 2014. Prevalência e classificações de lesões podais em bovinos leiteiros na região de Belo Horizonte-MG. Rev. Bras. Hig. San. Na. $8(1): 115-128$. 